\title{
REVISED EMI radiation of power transmission lines in Malaysia
}

\section{[version 2; peer review: 2 approved]}

\author{
Azhan Fikry1, Siow Chun Lim(D1, Mohd Zainal Abidin Ab Kadir² \\ ${ }^{1}$ Faculty of Engineering, Multimedia University, Cyberjaya, Selangor, 63100, Malaysia \\ ${ }^{2}$ Electrical \& Electronic Engineering, University Putra Malaysia, Serdang, Selangor, 43400, Malaysia
}

V2 First published: 10 Nov 2021, 10:1136

https://doi.org/10.12688/f1000research.73067.1

Latest published: 24 Feb 2022, 10:1136

https://doi.org/10.12688/f1000research.73067.2

\section{Abstract}

Background: There has been rising concern amongst the public regarding their home's proximity to high tension power transmission lines. The primary cause of fear is the impact of the electromagnetic interference (EMI) radiation on the nearby occupants' health. Despite the presence of national permissible limits of EMI radiation, there is still lack of information with regards to the EMI radiation of the types of power lines configuration in Malaysia.

Methods: The electric and magnetic fields of several selected power transmission lines were simulated using the EMFACDC software program from the recommendation ITU-T K.90. Five types of power transmission lines available in Malaysia are considered.

Results: It was found that the simulated electric and magnetic field levels at all the power lines' right of way (ROW) boundary complies with the prescribed exposure limit. However, the electromagnetic fields (EMF) level increases significantly as the separation distance is reduced from $30 \mathrm{~m}$. For a more conservative approach, the ROW can be set at $30 \mathrm{~m}$ across all transmission voltage level and corridor area condition.

Conclusion: It can be concluded that Malaysia's power transmission lines are within the prescribed exposure limits. To further minimize the electric and magnetic field level, it is recommended that the residential building should be built at least 30 meters away from the power transmission lines, especially for the $275 \mathrm{kV}$ double circuit, 275/132kV quadruple circuit, and 500kV double circuit lines.

Keywords

EMI radiation, power transmission lines, EMF, ELF, public exposure limit, Right-of-Way, ICNIRP

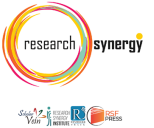

This article is included in the Research Synergy

Foundation gateway.
Open Peer Review

Approval Status

1 2

version 2

(revision)

$24 \mathrm{Feb} 2022$

version 1

10 Nov 2021
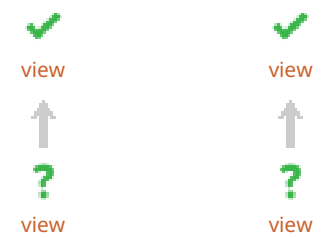

1. Jacek Paś, Military University of Technology, Warsaw, Poland

2. Muhammad Ammirrul Atiqi Mohd Zainuri (iD), Universiti Kebangsaan Malaysia, Bangi, Malaysia

Any reports and responses or comments on the article can be found at the end of the article. 


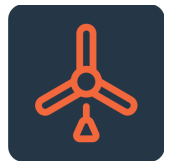

This article is included in the Energy gateway.

Corresponding author: Siow Chun Lim (chunlim87@gmail.com)

Author roles: Fikry A: Conceptualization, Formal Analysis, Methodology, Writing - Original Draft Preparation, Writing - Review \& Editing; Lim SC: Conceptualization, Supervision, Writing - Review \& Editing; Ab Kadir MZA: Conceptualization, Writing - Review \& Editing Competing interests: No competing interests were disclosed.

Grant information: The author(s) declared that no grants were involved in supporting this work.

Copyright: $\odot 2022$ Fikry A et al. This is an open access article distributed under the terms of the Creative Commons Attribution License, which permits unrestricted use, distribution, and reproduction in any medium, provided the original work is properly cited.

How to cite this article: Fikry A, Lim SC and Ab Kadir MZA. EMI radiation of power transmission lines in Malaysia [version 2; peer review: 2 approved] F1000Research 2022, 10:1136 https://doi.org/10.12688/f1000research.73067.2

First published: 10 Nov 2021, 10:1136 https://doi.org/10.12688/f1000research.73067.1 


\title{
REVISED Amendments from Version 1
}

Outline of the paper has been summarised at the end of the Introduction section.

Literature review has been expanded by relating contributions from previous articles to the research gap identified for this paper.

Additional paragraph to explain the flowchart in Figure 2 has been added in Method section.

Further discussions on Table 6 has been added.

Conclusion has been expanded.

Any further responses from the reviewers can be found at the end of the article

\author{
List of abbreviations \\ B: Magnetic Flux Density \\ CEU: The Council of The European Union \\ E: Electric Field Strength \\ EHV: Extra High Voltage \\ ELF: Extremely Low Frequency \\ EMF: Electromagnetic Fields \\ EMI: Electromagnetic Interference \\ EU: European Union \\ GHO: Global Health Observatory \\ ICNIRP: International Commission on Non-Ionizing Radiation Protection \\ IEEE: Institute of Electrical and Electronic Engineers \\ ITU: International Telecommunication Union \\ ITU-T: Telecommunication Standardisation Sector of International Telecommunication Union \\ MMU: Multimedia University \\ NTIS: National Technical Reports Library \\ ROW: Right-of-Way \\ T: Transposed Phasing \\ TNB: Tenaga Nasional Berhad \\ U: Untransposed Phasing \\ UK: United Kingdom \\ WHO: World Health Organization
}

\section{Introduction}

The public is getting increasingly concerned about the potential biological and health effects of power transmission lines considering the risk of exposure to electromagnetic interference (EMI) radiation. Biological effects are noticeable responses to a stimulus or an environmental change. ${ }^{1}$ At an extreme level, electromagnetic fields (EMF) will affect humans' health, such as micro shocks and induced currents in the body. ${ }^{2}$ Power transmission lines produce electric and magnetic fields at an extremely low frequency. These transmission lines were sometimes located in close proximity to residential areas, which could potentially increase the exposure level of electromagnetic radiation. Although national and international guidelines have established exposure limits to protect the public against high-level EMF that might be harmful, there are still rising concerns among the public on whether their residency is affected by such radiation, especially to those living near power transmission lines. ${ }^{3}$

Overhead power transmission lines in Malaysia are typically rated at $132 \mathrm{kV}, 275 \mathrm{kV}$, and $500 \mathrm{kV}$. Figure 1 shows a typical dimension for various power transmission lines towers in Malaysia. These dimensions agree well with the transmission line design manual in I. Beck. ${ }^{4}$

Several isolated simulation studies on the propagation of EMF around the $132 \mathrm{kV}, 275 \mathrm{kV}$, and $500 \mathrm{kV}$ power transmission lines in Malaysia has been presented in Sahbudin et al., ${ }^{5}$ Said and Hussain, ${ }^{6}$ Jimbin and Ahmad, ${ }^{7}$ and Rahman et al. ${ }^{8}$ All of the studies referenced here are related to EMF propagation around the power transmission lines and how it affects the nearby public. Sahbudin et al. ${ }^{5}$ have analyzed and computed the amount of electrical potential and EMF level at a specific point and distance from Malaysia's $132 \mathrm{kV}$ and $275 \mathrm{kV}$ double circuit power transmission lines. On the other hand, Said and Hussain, ${ }^{6}$ together with Jimbin and Ahmad, ${ }^{7}$ conducted magnetic field simulation studies on the quadruple circuit power transmission lines in Malaysia. Rahman et al. ${ }^{8}$ has presented the magnetic field radiation simulation studies at multiple locations in Malaysia, accounting for the $132 / 132 \mathrm{kV}$ quadruple circuit and the $500 \mathrm{kV}$ double circuit power transmission lines. The studies were conducted in several locations in Malaysia in which the EMF level was analyzed and computed at a 


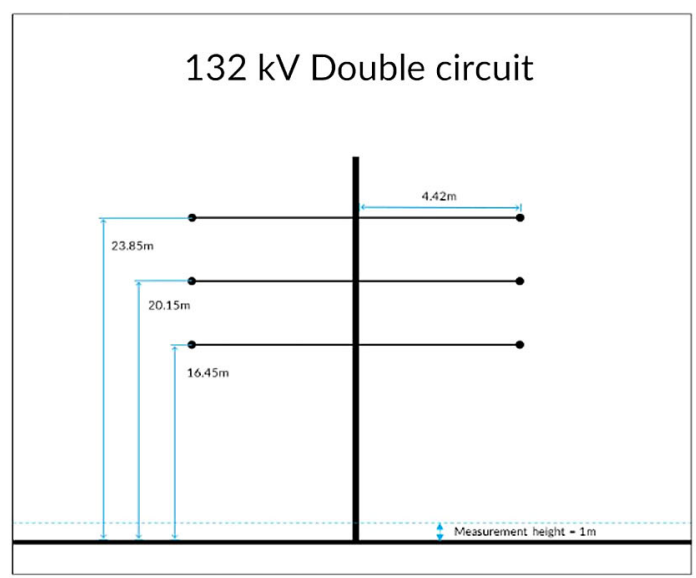

275/132 kV Quadruple circuit
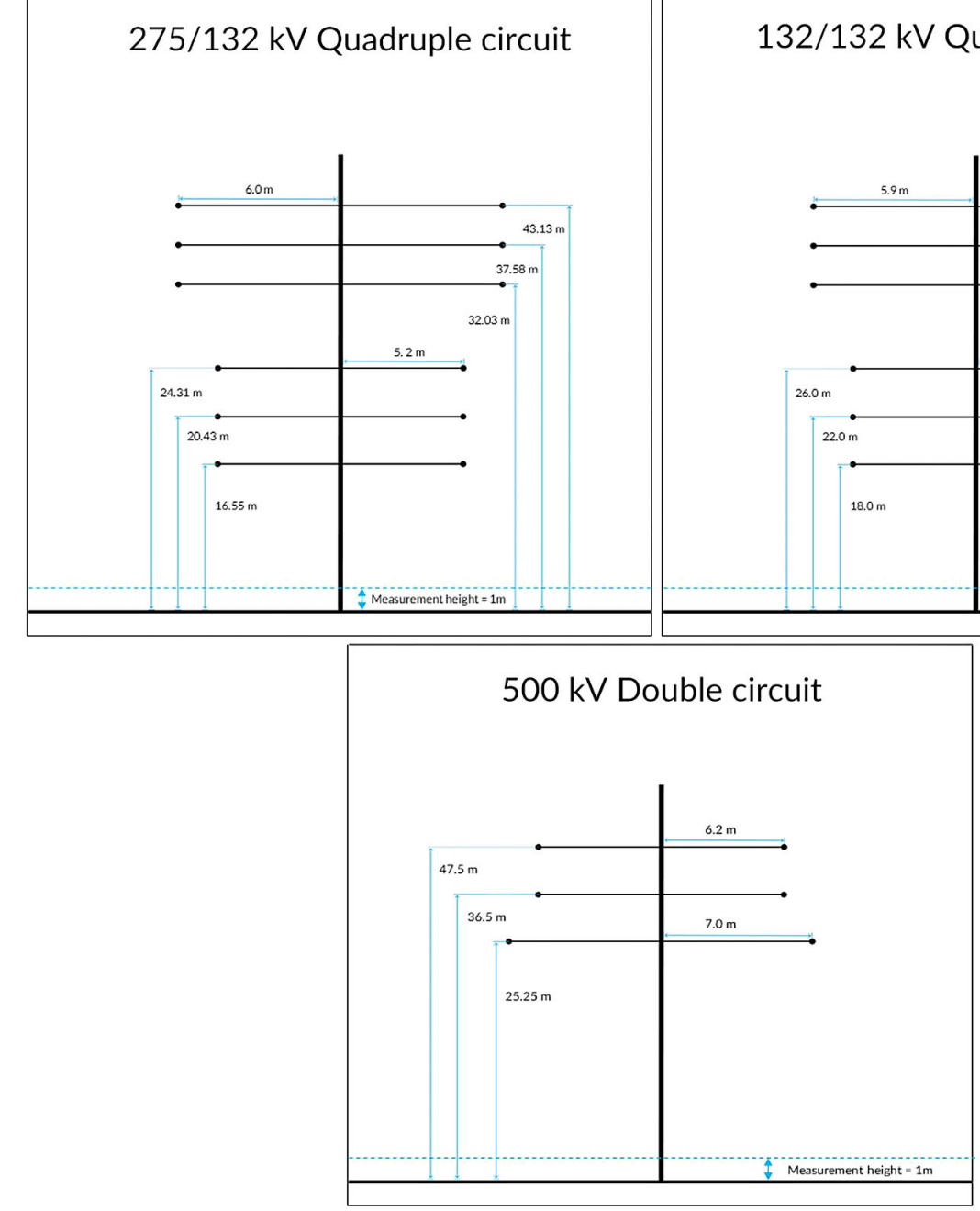

Figure 1. Overhead power transmission lines towers in Malaysia. ${ }^{4-6,10,11}$

specific point and distance from the power transmission line. However, analysis of the compliance of the EMF with recommended public exposure limits remains lacking as no specific study evaluated the EMF level from every type of power transmission line in Malaysia. Hence, this paper presents the simulations of the electromagnetic field radiation from various high-voltage power transmission lines in Malaysia, conducted simultaneously to provide an overall comparison in terms of their corresponding EMF level as a function of distance away from the power lines. The simulated EMF levels are 
then benchmarked with public exposure limits recommended by international standards and the commenced right of way (ROW), after which the minimum safe distance of power transmission lines from residential areas can then be determined.

This paper is organized in the following manner. The methods section explains the overall procedures and techniques involved in identifying, processing, and analyzing information regarding the EMI radiation of power transmission lines to implement the research objective. Then the results section presents the collected data of the EMF value of the power transmission lines relative to the selected exposure limits and commenced ROW. This paper ends with a conclusion, which synthesizes the key points from the collected data and benchmarked results, a safer approach to minimize the exposure to EMF from the power transmission lines in Malaysia is also recommended.

\section{Methods}

Figure 2 depicts the overall project flow for this work. ${ }^{20}$ Initially, EMF from the power transmission lines was calculated and then simulated using the existing source (EMFACDC program). Both calculation and simulation results are then compared to test for the accuracy of the simulation program. If the results are satisfactory, the simulations of EMF from power transmission lines were conducted again using the collected parameter of the power lines in Malaysia. The EMF of

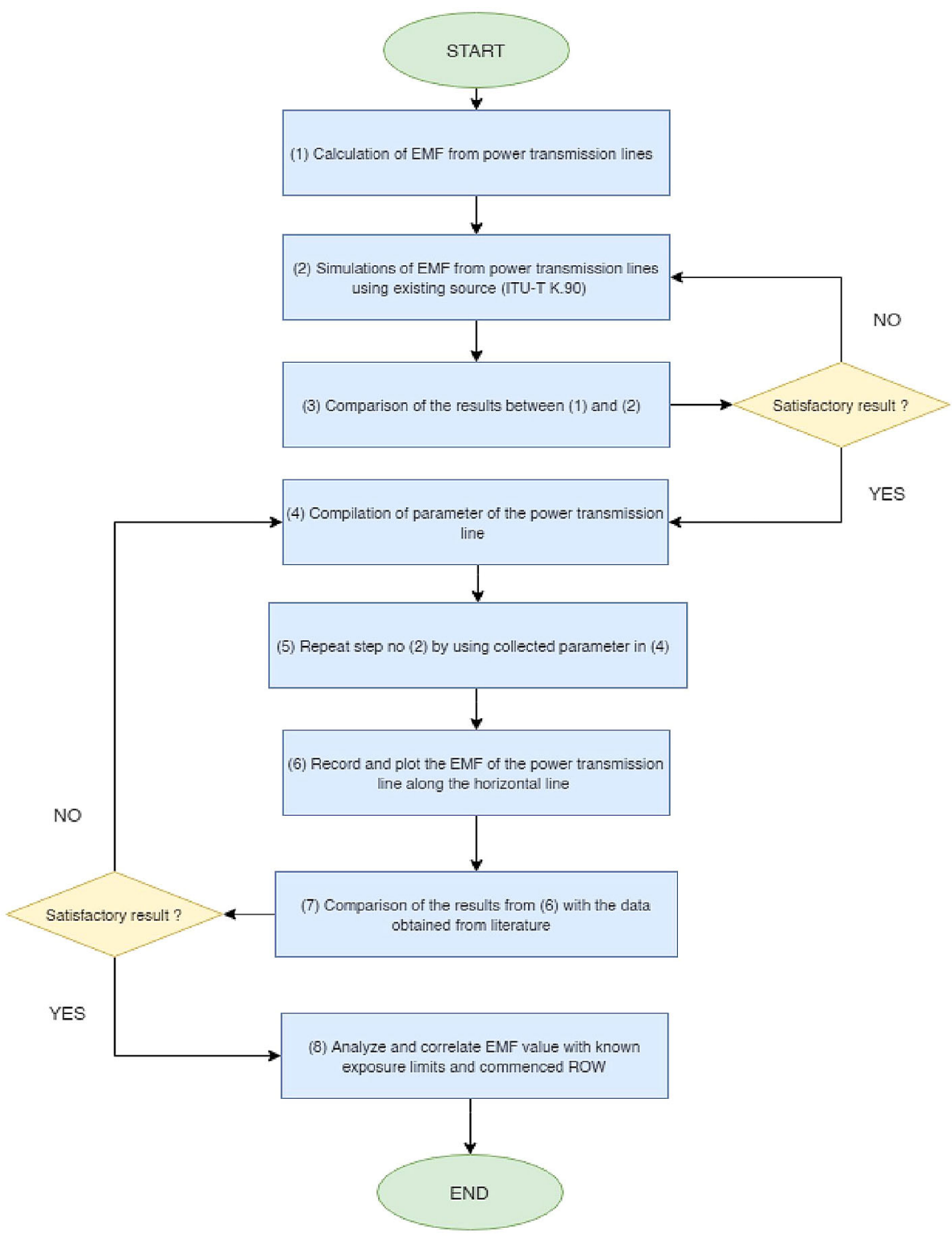

Figure 2. Overall project flow. 


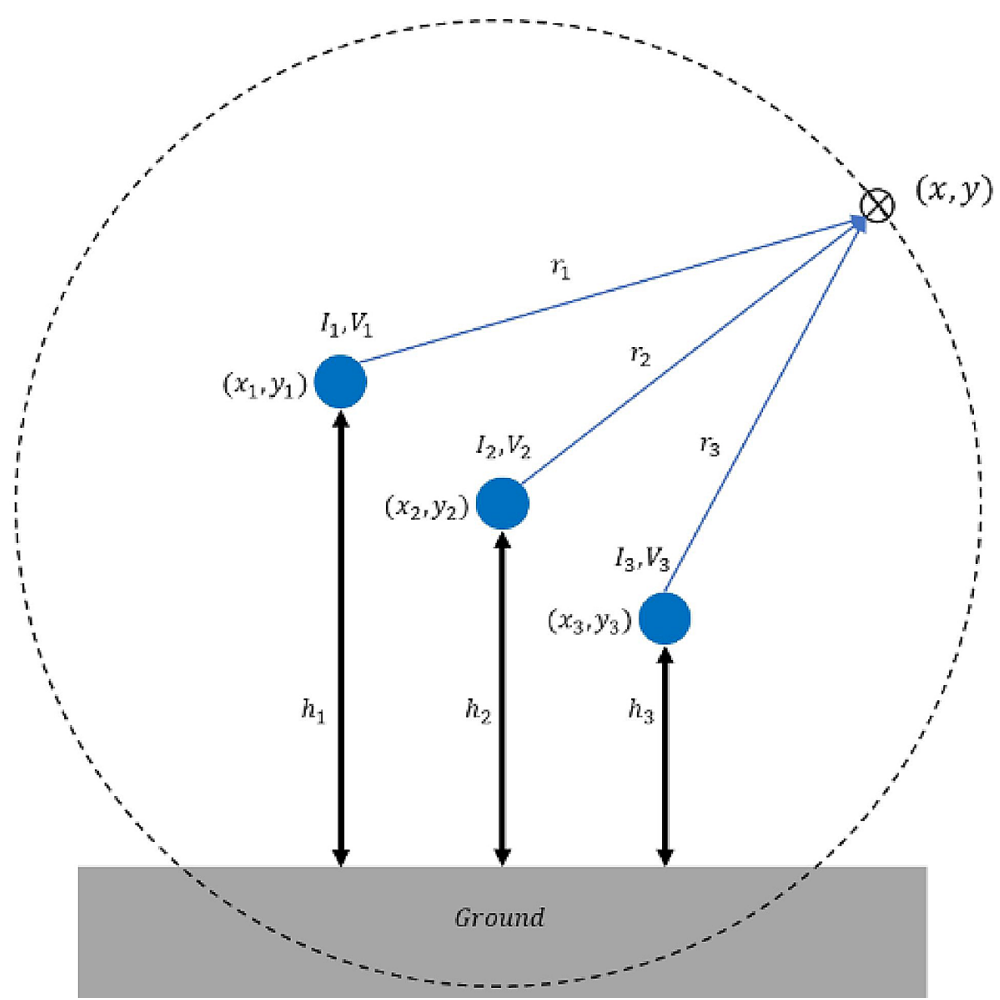

Figure 3. Geometrical illustration for the magnetic and electric field calculation. ${ }^{12}$

the power transmission line along the horizontal line is then plotted and recorded. The EMF value is then verified by comparing it with the data obtained from the literature. If the results are satisfactory, the EMF value from power transmission lines are analyzed and correlated with the known exposure limits and commenced ROW. The field calculation for a three-phase circuit power transmission line is based on the principle of superposition. ${ }^{9,12}$ Suppose the three conductors are labelled with an index $k$ that ranges from one to three. For each conductor, there is a combination of complex current $I_{k}$ and complex voltage $V_{k}$. The position of the conductors is denoted by $\left(x_{k}, y_{k}\right)$ in which the origin can be chosen at any point. The objective is to calculate the field at a point of interest $(x, y)$. Every conductor is above the earth at the height of $h_{k}$ and $r_{k}$ away from the point of interest as shown in Figure 3. For a single conductor-carrying current $I$ along a straight line, the magnetic field magnitude, $B$ is given by:

$$
B=\frac{\mu_{o} I_{o}}{2 \pi\left(x^{2}+y^{2}\right)^{\frac{1}{2}}}
$$

where $\mu_{o}$ : permittivity of free space.

The resultant magnetic field due to a three-phase conductor system is the complex summation of contributing magnetic field vectors. The vertical and horizontal components of $B$ can then be established geometrically as shown in the National Grid EMF guide. ${ }^{13}$ Each conductor is then resolved into an in-phase and out-of-phase component to determine the current relation. This is done by referring to the phasor diagram depicted in the National Grid EMF guide. ${ }^{13}$ By referring to the phasor diagram, all the in-phase and out-of-phase components can be extracted using the sin and $\cos$ of $30^{\circ}\left(120^{\circ}-90^{\circ}\right)$. For a single three-phase circuit, it does not matter which order these conductors were placed. Each conductor will have a phase $120^{\circ}$ apart from the other two conductors. Being $120^{\circ}$ apart makes the phases balanced in which the power transfer is constant at any point. This method has also been used in other isolated simulation studies presented in Refs. 6 and 7. For more than one circuit, a consistent convention should be applied when defining the phases to achieve optimum efficiency as shown in the National Grid EMF guide. ${ }^{13}$

By multiplying both geometrical and current term, four components of the field can be obtained, which is $B_{i n, x}, B_{i n, y}$, $B_{\text {out }, x}$, and $B_{\text {out }, y}$. The total magnetic field is then given by: 


$$
B_{\text {total }}=\sqrt{\left(B_{\text {in }, x}\right)^{2}+\left(B_{\text {in }, y}\right)^{2}+\left(B_{\text {out }, x}\right)^{2}+\left(B_{\text {out }, y}\right)^{2}}
$$

The electric field generated by a system of conductors is attributable to the potential $V_{k}$ applied to each of them. The electric coupling between conductors should be considered as well in order to determine the linear charge density. ${ }^{12}$ The potential $\mathrm{V}$ at its boundary surface for a single conductor of effective radius $\mathrm{R}$ ' at a height $h$ above the ground is given by:

$$
V=\frac{\lambda}{2 \pi \varepsilon_{0}} \ln \frac{2 h}{R^{\prime}}
$$

where $\lambda$ : equivalent density of charge; $\varepsilon_{0}$ is the permittivity of free space.

The conductor's electrical image's contribution was also incorporated in equation 3 in the case of perfectly conducting ground. For a known value of $V$, the equivalent linear density of charge can be assessed along with its electrical image, which is then used to calculate the electric field vector at any point of interest (x,y). A single contribution to the electric field either from the conductor or its electrical image is given by:

$$
\bar{E}=\frac{\lambda}{2 \pi \varepsilon_{0}} \frac{1}{r} \hat{r}
$$

where $r$ : distance from the conductor (or image) to the point of interest; $\hat{r}$ is the respective unit vector. In the event of multiple conductors (three conductors for a three-phase circuit), the equivalent linear density of charge is determined by solving the linear system of equations given by:

$$
[P][\lambda]=[V]
$$

where $[P]$ : coupling matrix; $[\lambda]$ : equivalent densities vector; $[V]$ : vector of potentials applied to the conductors.

The coupling matrix can be defined as:

$$
P_{i i}=\frac{1}{2 \pi \varepsilon_{0}} \ln \frac{2 h_{i}}{R_{i}} \quad P_{i j}=\frac{1}{2 \pi \varepsilon_{0}} \ln \frac{D_{i j}}{d_{i j}}
$$

Here the conductor indexes are denoted by $i j$ from 1 to $N$. $D_{i j}$ represents the distance between the conductor $i$ and the image of the conductor $j$ while $d_{i j}$ represents the distance between the conductor $i$ and the conductor $j$. With $V$ as the voltage level of the overhead power transmission lines in Malaysia as depicted in Figure 1, the charge density can be obtained by inverting the matrix equation above where:

$$
[\lambda]=[V][P]^{-1}
$$

After solving the system of equations, the corresponding charge densities are then used to calculate the resultant electric field at the point of interest, as in the case of a single conductor described above. The resulting electric field due to a system of conductors (and their electric images) is obtained by the complex summation of the contributing electric field vectors.

In this study, the EMF simulations were conducted using the publicly available EMFACDC v2.0, update to Appendix II program developed by the Telecommunication Standardization Sector of International Telecommunication Union (ITU-T) under the series of recommendations known as Series K (https://www.itu.int/rec/T-REC-K.90-201905-I! Amd1/en). Table 1 shows the geometrical parameters of this study.

Verification of the EMFACDC program is performed by comparing the simulation value obtained from the program with the value obtained through manual calculation performed for a typical power line setup. To do this, the exact parameter of the selected power line is keyed into the program. The results were considered satisfactory if both of the values were approximately similar to each other. This study chooses the magnetic field level of a typical United Kingdom (UK) $400 \mathrm{kV}$ double circuit power transmission line at 10 meters from the centerline. As this verification purpose is to test for the accuracy of the simulation program, any type of power line is applicable.

Rated current is used for each conductor in the power lines to test for the maximum current value possible as applied in Jimbin and Ahmad. ${ }^{7}$ The current in the left and right circuits are assumed to be equal in order to ensure the EMF level is 
Table 1. Geometrical parameters.

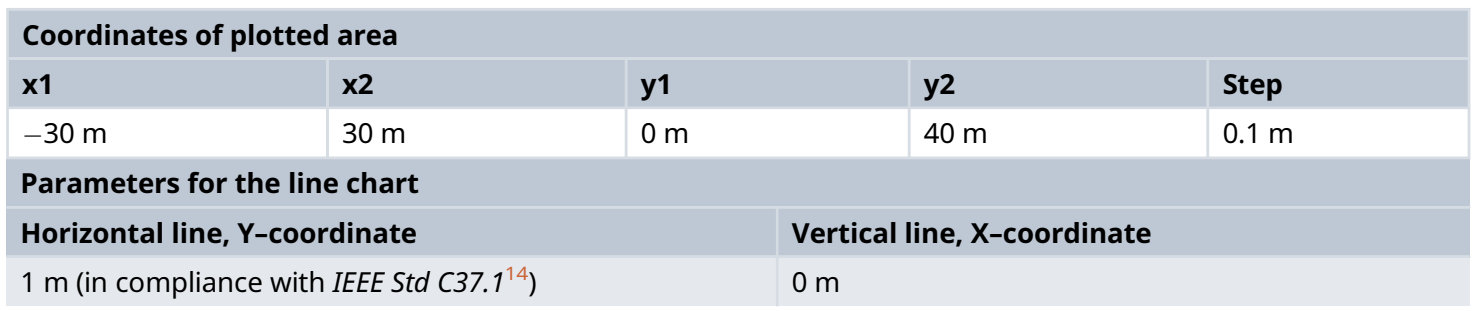

balanced at the left and right ROW boundary. For each type of power transmission line, the conductors are represented by their phase pattern and assigned their own phase code. Standard transposed phasing and un-transposed phasing will be considered in this study as shown in Figures 4 and 5. Transposition of phasing is performed to reduce crosstalk or interference which arises by current flowing in the conductors. Therefore, un-transposed phasing is expected to produce the highest magnetic flux density and vice versa, as suggested in Said et al., ${ }^{6}$ under Group 5 conductor phase pattern. Although transposed phasing is used by most of the power transmission lines in the National Grid to significantly reduce the magnetic and electric field generated, un-transposed phasing is still considered in this study as it is not always feasible to transpose every line.

The parameters for each type of power lines which are based on Malaysia's specific power transmission line were consolidated from R. K. Z. Sahbudin et al., ${ }^{5}$ I. Said et al., ${ }^{6}$ V. S. Jimbin and Ahmad, ${ }^{7}$ N. A. Rahman et al., ${ }^{8}$ I. M. Rawi et al., ${ }^{10}$ and N. H. N. Hassan et al. ${ }^{11}$ These dimensions also agree well with the Transmission Line Design Manual prepared by R. W. Beck, Inc., for the use of Tenaga Nasional Berhad (TNB) in June 2000. ${ }^{4}$ Five types of power transmission lines available in Malaysia are considered in this study. These are the power transmission lines typically used in Malaysia, known with voltage levels of $132 \mathrm{kV}, 275 \mathrm{kV}$, and $500 \mathrm{kV}$ :

1. $132 \mathrm{kV}$ double circuit power transmission line.

2. $275 \mathrm{kV}$ double circuit power transmission line.

3. $132 / 132 \mathrm{kV}$ quadruple circuit power transmission line.

4. $275 / 132 \mathrm{kV}$ quadruple circuit power transmission line.

5. $500 \mathrm{kV}$ double circuit power transmission line.
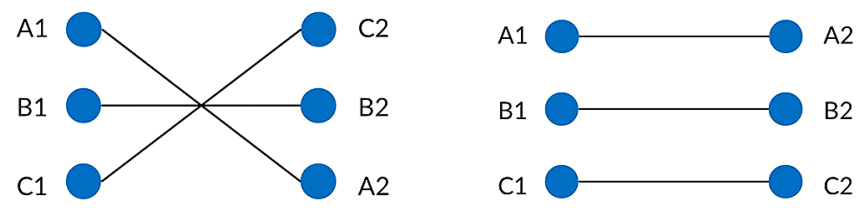

Figure 4. Double circuit phase configuration for standard transposed phasing (left) and un-transposed phasing (right).
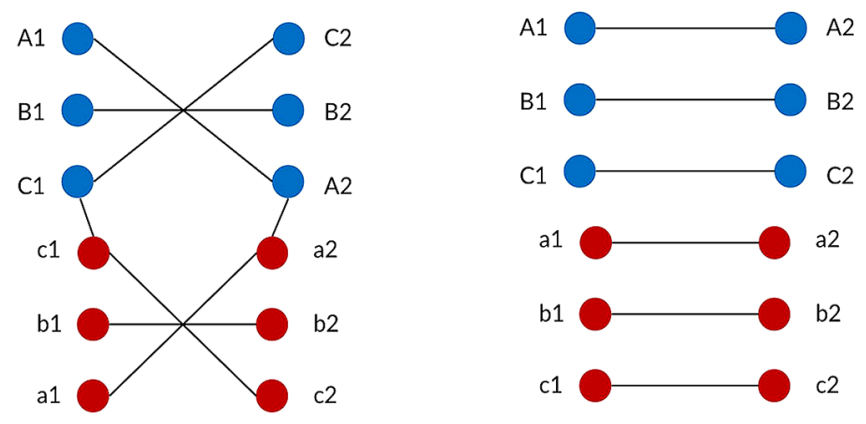

Figure 5. Quadruple circuit phase configuration for standard transposed phasing (left) and un-transposed phasing (right). 
These power transmission lines were identified based on the Suruhanjaya Tenaga Wayleave for Electricity Supply Lines. ${ }^{15}$ They were chosen as they were categorized under high voltage and extra-high voltage distribution and ran under three-phase configurations. Tables 2 and 3 show samples of the input data used for the field simulation of $275 / 132 \mathrm{kV}$ quadruple circuit power line and $500 \mathrm{kV}$ double circuit power lines in Malaysia. ${ }^{7,8,10}$ The remaining set of input data i.e. of $132 \mathrm{kV}$ double circuit power line, $275 \mathrm{kV}$ double circuit power line and 132/132 kV quadruple circuit power line is made available in Tables 3,4 , and $5 .{ }^{20}$

Table 2. 275/132 kV quadruple circuit power line.

\begin{tabular}{|c|c|c|c|c|c|c|c|c|}
\hline \multirow[t]{2}{*}{ No } & \multicolumn{2}{|c|}{ Phase code } & \multicolumn{2}{|c|}{ Cond. coordinate } & \multirow[t]{2}{*}{ Current rating $(\mathrm{A})$} & \multirow[t]{2}{*}{ Voltage level (kV) } & \multicolumn{2}{|c|}{ Phase angle (Deg.) } \\
\hline & $\mathbf{U}$ & $\mathbf{T}$ & $x(m)$ & $y(m)$ & & & $\mathbf{U}$ & $\mathbf{T}$ \\
\hline 1 & A1 & $\mathrm{A} 1$ & -6.0 & 43.13 & 1232 & 275 & 0 & 0 \\
\hline 2 & B1 & B1 & -6.0 & 37.58 & 1232 & 275 & 120 & 120 \\
\hline 3 & C1 & $\mathrm{C} 1$ & -6.0 & 32.03 & 1232 & 275 & 240 & 240 \\
\hline 4 & A2 & $\mathrm{C} 2$ & 6.0 & 43.13 & 1232 & 275 & 0 & 240 \\
\hline 5 & B2 & B2 & 6.0 & 37.58 & 1232 & 275 & 120 & 120 \\
\hline 6 & $\mathrm{C} 2$ & $\mathrm{~A} 2$ & 6.0 & 32.03 & 1232 & 275 & 240 & 0 \\
\hline 7 & a1 & c1 & -5.2 & 24.31 & 729 & 132 & 0 & 240 \\
\hline 8 & b1 & b1 & -5.2 & 20.43 & 729 & 132 & 120 & 120 \\
\hline 9 & c1 & a1 & -5.2 & 16.55 & 729 & 132 & 240 & 0 \\
\hline 10 & a2 & a2 & 5.2 & 24.31 & 729 & 132 & 0 & 0 \\
\hline 11 & b2 & b2 & 5.2 & 20.43 & 729 & 132 & 120 & 120 \\
\hline 12 & c2 & c2 & 5.2 & 16.55 & 729 & 132 & 240 & 240 \\
\hline
\end{tabular}

Table 3. 500 kV double circuit power line.

\begin{tabular}{|l|l|l|l|l|l|l|l|l|}
\hline No & \multicolumn{3}{|c|}{ Phase code } & \multicolumn{2}{|c|}{ Cond. coordinate } & Current rating (A) & Voltage level (kV) & \multicolumn{2}{|c|}{ Phase angle (Deg.) } \\
\hline & $\mathbf{U}$ & $\mathbf{T}$ & $\mathbf{x ( m )}$ & $\mathbf{y}(\mathbf{m})$ & & & $\mathbf{U}$ & $\mathbf{T}$ \\
\hline 1 & A1 & A1 & -6.2 & 47.5 & 2309.4 & 500 & 120 & 120 \\
\hline 2 & B1 & B1 & -6.2 & 36.5 & 2309.4 & 500 & 240 & 240 \\
\hline 3 & C1 & C1 & -7.0 & 25.25 & 2309.4 & 500 & 0 & 240 \\
\hline 4 & A2 & C2 & 6.2 & 47.5 & 2309.4 & 500 & 120 & 120 \\
\hline 5 & B2 & B2 & 6.2 & 36.5 & 2309.4 & 500 & 240 & 0 \\
\hline 6 & C2 & A2 & 7.0 & 25.25 & 2309.4 & 500 & & 0 \\
\hline
\end{tabular}

Table 4. Benchmarked public exposure limits.

\begin{tabular}{|c|c|c|}
\hline No & Category & Public exposure limit \\
\hline 1. & Specific International Organization & $\begin{array}{l}\text { ICNIRP } 2010^{16} \\
\text { (5 kV/m for electric field and } \mathbf{2 0 0} \boldsymbol{\mu} \mathbf{T} \text { for magnetic field) }\end{array}$ \\
\hline 3. & Regional variation of a country & $\begin{array}{l}\text { Exposure limit for the residential region - Slovenia and Italy }{ }^{18} \\
(\mathbf{0 . 5} \mathbf{~ k V / m} \text { for electric field and } \mathbf{1 0} \boldsymbol{\mu} \mathbf{T} \text { for magnetic field) }\end{array}$ \\
\hline
\end{tabular}




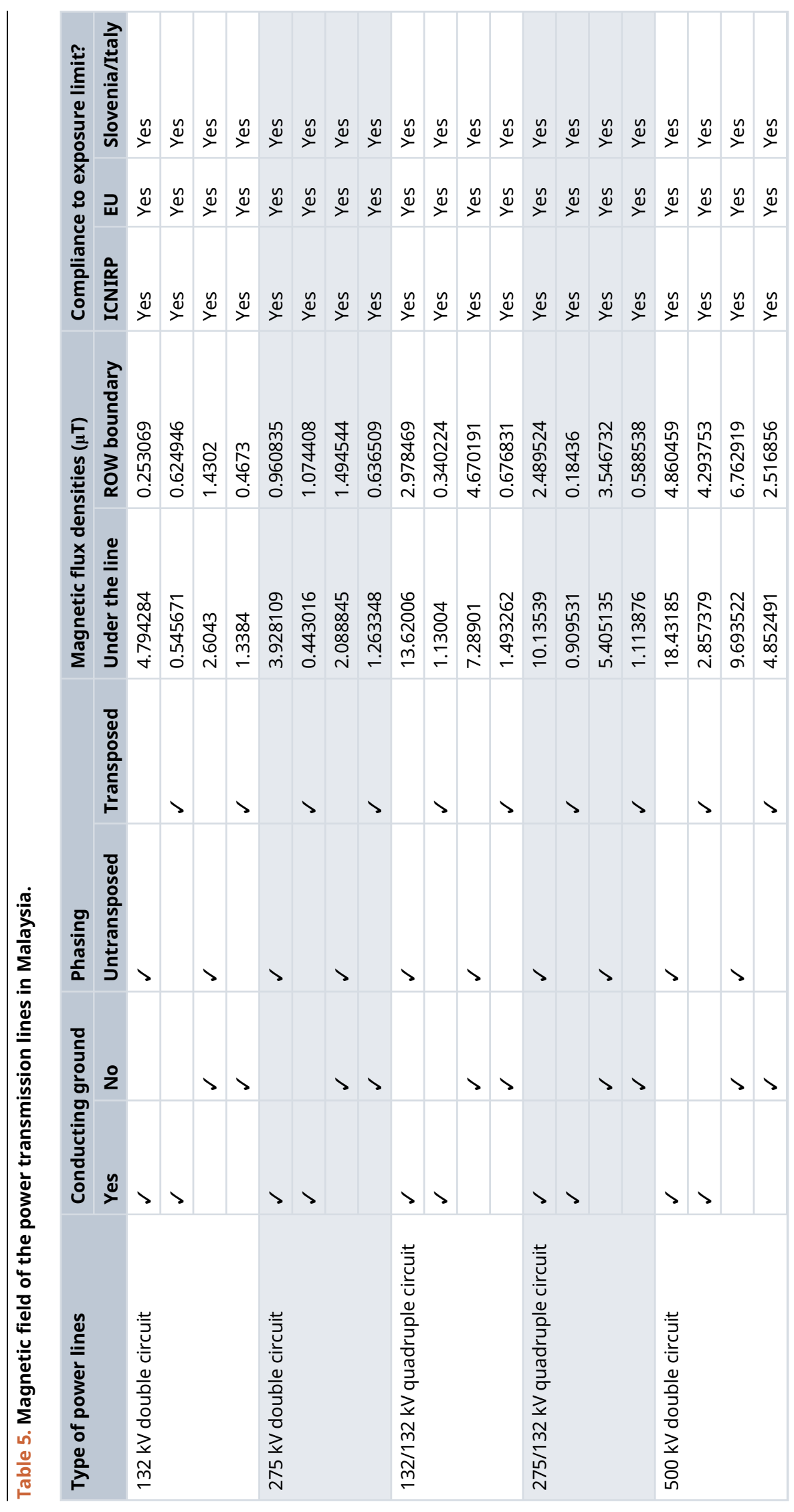




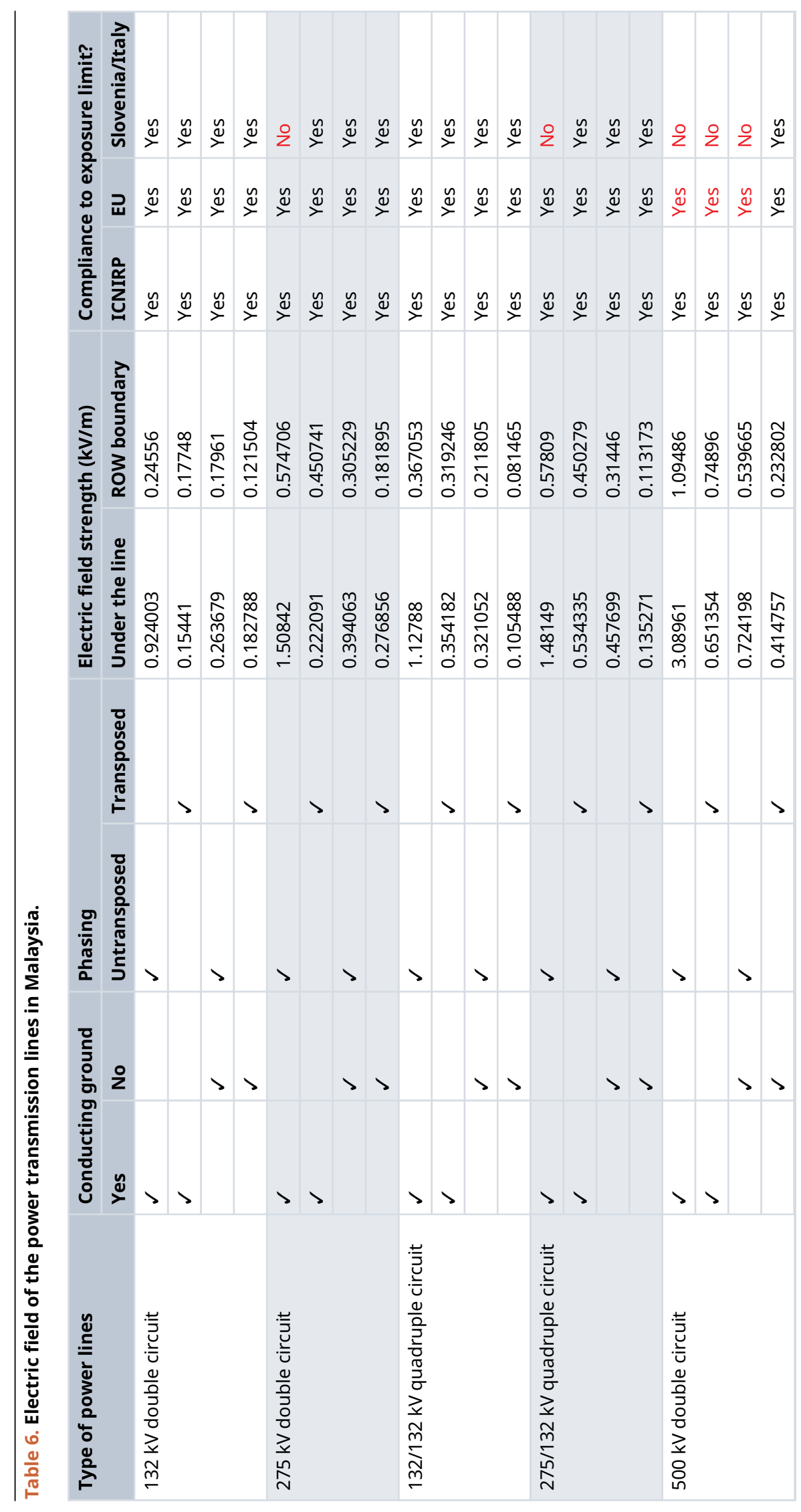


The simulation of electric and magnetic fields at various horizontal distance from the center of power lines was analyzed and benchmarked with public exposure limits from selected organizations defined in Table 4 (Table $8^{20}$ ). The International Commission on Non-Ionizing Radiation Protection (ICNIRP) and European Union (EU) limits were selected as they were used in most of the countries globally while Slovenia and Italy were purposely selected due to their more stringent requirement. In this study, public exposure limits from three different categories are chosen for the correlation studies to satisfy all variants in every country. The exposure limits selected were used to prevent established effects at high electromagnetic field level. The simulation is also benchmarked with the commenced ROW from Suruhanjaya Tenaga for each type of power lines to estimate the electromagnetic field level at the minimum separation distance from the center of power lines. ${ }^{15}$ The ROW specified by the Suruhanjaya Tenaga 15 is applicable for the correlation process as it is the currently commenced minimum safe distance for power transmission lines in Malaysia. Correlation between the exposure limit and the commenced ROW is analyzed by observing the electromagnetic field level at the minimum separation distance. The field level at the minimum separation distance is considered to be safe if it is lower than the exposure limits.

\section{Results}

The simulation was initially verified with a typical setup of a double circuit power transmission line in the United Kingdom ${ }^{19}$ as outlined in the methods section (Table $2^{20}$ ). Upon verification, simulation of magnetic flux densities and the electric field strength from different types of power lines at the horizontal line were conducted. The magnetic and electric field of the power transmission lines relative to the selected exposure limits and commenced ROW were summarized and shown in Tables 5 and 6 (Table 10 and Table $11^{20}$ ). The average EMF values recorded for each power lines at ROW boundary were also portrayed in Table $12 .{ }^{20}$ In this paper, EMI radiation refers to the disturbance caused by the electric and magnetic field towards a subject. The probability of EMI occurring depends highly on the electric and magnetic field strength. ${ }^{21}$

The magnetic field due to all the power transmission lines are within the exposure limits set by the organizations in Table 4 (Table $8^{20}$ ). The highest magnetic flux densities at the ROW boundary were simulated from the un-transposed $500 \mathrm{kV}$ double circuit power line under non-conducting ground condition, while the lowest was simulated from the transposed $275 / 132 \mathrm{kV}$ quadruple circuit under the conducting ground condition, as shown in Table $10 .{ }^{20}$ Even directly below the overhead power lines, the magnetic field levels are still within the exposure limit recommended by ICNIRP and EU. ${ }^{16,17}$

Similarly, the electric field due to all the power transmission lines are within with the exposure limits set by ICNIRP and EU. However, $25 \%$ of the power transmission lines exceed the stricter exposure limit practiced in Slovenia and Italy. The highest electric field strength data at the ROW boundary was simulated from the $500 \mathrm{kV}$ double circuit power line under un-transposed and conducting ground condition, while the lowest was simulated from the $132 / 132 \mathrm{kV}$ quadruple circuit under transposed and non-conducting ground (Table $11^{20}$ ). Similar to the magnetic field simulation, even below the overhead power lines, the electric field levels are still within the exposure limit recommended by ICNIRP 2010 and EU Recommendation 1999. ${ }^{16,17}$ The transposed conductors give significantly lower magnetic flux densities and electric field strength.

\section{Conclusions}

The electric and magnetic fields due to five types of power transmission lines in Malaysia were simulated and benchmarked with the known exposure limits and commenced ROW. The electric and magnetic field level for all types of power lines at the edge of ROW was found to be lower than the exposure limit recommended by ICNIRP. Moreover, the average EMF values recorded for each power line at the ROW boundary were determined to be significantly lower than the ICNIRP recommendation. The substantial difference between the simulated values of EMF due to power transmission lines in Malaysia at the ROW boundary with the exposure limit recommended by ICNIRP is noticeable by referring to the output data representations of this study. Hence, it can be concluded that Malaysia's power transmission lines are within the safe exposure limit recommended by ICNIRP. To further minimize the electric and magnetic field level, it is recommended that the residential building should be built at least 30 meters away from the power transmission lines, especially for the $275 \mathrm{kV}$ double circuit, $275 / 132 \mathrm{kV}$ quadruple circuit, and $500 \mathrm{kV}$ double circuit lines. It is also recommended that Malaysia's regulatory bodies develop and implement regional exposure limits to specific locations where the public could potentially experience long-term exposure to EMF radiation, such as in the residential building, school, and hospital. Utilizing a proper phase arrangement was also crucial in the power transmission line's design process as it could reduce the EMF radiation.

\section{Software availability}

The software EMFACDC v2.0, appendix 1 (2020) which is used in simulating the electric and magnetic field in this study is publicly available to download at https://www.itu.int/rec/T-REC-K.90-201905-I!Amd1/en. 


\section{Author roles}

Azhan F.: Conceptualization, Formal Analysis, Methodology, Writing - Original Draft Preparation, Writing - Review \& Editing;

Siow C.L.: Conceptualization, Supervision, Writing - Review \& Editing

M. Zainal A.A.K.: Conceptualization, Writing - Review \& Editing

\section{Data availability statement}

Underlying data

Figshare: Data for Electric and Magnetic Field of Power Transmission Lines in Malaysia.

https://doi.org/10.6084/m9.figshare.16577423.v2. ${ }^{20}$

The project contains the following underlying data:

- Data for Electric and Magnetic Field of Power Transmission Lines in Malaysia. (Simulated dataset of electric and magnetic field of five types of power transmission lines in Malaysia in Tables 1-12 and Figures 1-20).

- Figure 1. (Overhead power transmission lines towers in Malaysia).

- Figure 2. (Overall project flow).

- Figure 3. (Geometrical illustration for the magnetic and electric field calculation).

- Figure 4. (Double circuit phase configuration for standard transposed phasing (left) and un-transposed phasing (right).

- Figure 5. (Quadruple circuit phase configuration for standard transposed phasing (left) and un-transposed phasing (right).

Data are available under the terms of the Creative Commons Zero "No rights reserved" data waiver (CC0 1.0 Public domain dedication).

\section{Acknowledgements}

The authors would like to thank the International Telecommunication Union (ITU) for providing the EMFACDC v2.0, appendix 1 (2020) software and the Faculty of Engineering, Multimedia University (MMU) for supporting this study.

1. World Health Organization (WHO): Radiation: Electromagnetic fields. Aug. 04, 2016. (Accessed Feb. 10, 2021).

Reference Source

2. Department of Energy \& Climate Change: Power Lines: Control of Microshocks and other indirect effects of public exposure to electric fields A voluntary Code of Practice. no. July, 2013.

3. Farag AS, Cheng TC, Penn D: Development of an electromagnetic fields risk communication plan. Electr. Power Syst. Res. 1999; 50(1): 55-63.

Publisher Full Text

4. U.S. Department of Commerce: I. Beck (RW), "Tenaga Nasional Berhad Transmission Line Design Manual. Final Submittal.| National Technical Reports Library - NTIS. National Technical Reports Library. 2000. (accessed Feb. 11, 2021) Reference Source

5. Sahbudin RKZ, Fauzi SA, Hitam S, et al.: Investigation of electrical potential and electromagnetic field for overhead high voltage power lines in Malaysia. J. Appl. Sci. 2010; 10(22):
2862-2868

Publisher Full Text

6. Said I, Hussain HB: Computation of magnetic field from quadruple tower transmission lines in Malaysia. Proc. Univ. Power Eng. Conf. 2008. Publisher Full Text

7. Jimbin VS, Ahmad NA: Magnetic field measurement from 132/275 kV overhead power lines. J. Teknol. Jan. 2017; 79(1): 89-95. Publisher Full Text

8. Rahman NA, Mahadi WNL, Said I, et al.: Simulation Approach in Evaluating the Electromagnetic fields from the new lines of Extra High Voltage (EHV) circuits near residential area in Malaysia. Eur. J. Sci. Res. 2010; 40(2): 189-198.

9. Ismail HM: Characteristics of the magnetic field under hybrid ac/dc high voltage transmission lines. Electr. Power Syst. Res. 2009; 79(1): 1-7. Publisher Full Text

10. Rawi IM, Abidin Ab Kadir MZ, Gomes C, et al:: A case study on 500 kV line performance related to lightning in Malaysia. IEEE Trans. 
Power Deliv. Oct. 2018; 33(5): 2180-2186. Publisher Full Text

11. Hassan NHN, Bakar AHA, Mokhlis $\mathrm{H}$, et al.: Analysis of arrester energy for $132 \mathrm{kV}$ overhead transmission line due to back flashover and shielding failure. PECon 2012-2012 IEEE Int. Conf Power Ener. 2012; 1(December): 683-688. Publisher Full Text

12. International Telecommunication Union (ITU): ITU-T Evaluation techniques and working procedures for compliance with exposure limits of network operator personnel to power-frequency electromagnetic fields Recommendation ITU-T K.90. 2018. Reference Source

13. National Grid EMF: How to calculate the magnetic field from a threephase circuit. (accessed Feb. 11, 2021). Reference Source

14. IEEE: IEEE Std C37.1 - Standard Definition, Specification, and Analysis of Systems Used for Supervisory Control, Data Acquisition, and Automatic Control. 1994; vol. 1994

15. Energy Commission of Malaysia: Wayleave for Electricity Supply Lines: Your Right and Safety. 2014.

16. International Commission on Non-Ionizing Radiation Protection (ICNIRP): ICNIRP Publication 2010, "ICNIRP Guidelines for Limiting Exposure to Time-Varying Electric and Magnetic Fields
(1 Hz-100 kHz) International Commission on Non-Ionizing Radiation Protection*,". 2010

Publisher Full Text

17. The Council Of The European Union (CEU): Council

Recommendation of 12 July 1999 on the limitation of exposure of the general public to electromagnetic fields $(0 \mathrm{~Hz}$ to $300 \mathrm{GHz})$. Off. J. Eur. Communities. 1999; 59-70.

18. World Health Organization (WHO):GHO|By category|Exposure limits for low-frequency fields (public) - Data by country. WHO.

19. National Grid EMF: Geometries of power lines. (accessed Feb. 11, 2021).

Reference Source

20. Chun Lim S, Fikry A, Ab-Kadir MZA: Data for Electric and Magnetic Field of Power Transmission Lines in Malaysia. figshare. Dataset. 2021.

Publisher Full Text

21. Driessen S, Napp A, Schmiedchen $\mathrm{K}$, et al.: Electromagnetic interference in cardiac electronic implants caused by novel electrical appliances emitting electromagnetic fields in the intermediate frequency range: a systematic review. Europace. 2019; 21(2): 219-229.

Publisher Full Text 


\section{Open Peer Review}

\section{Current Peer Review Status:}

\section{Version 2}

Reviewer Report 22 March 2022

https://doi.org/10.5256/f1000research.121539.r125173

(C) 2022 Mohd Zainuri M. This is an open access peer review report distributed under the terms of the Creative Commons Attribution License, which permits unrestricted use, distribution, and reproduction in any medium, provided the original work is properly cited.

\section{Muhammad Ammirrul Atiqi Mohd Zainuri}

Electrical, Electronic and Systems Engineering, Faculty Engineering and Built Environment, Universiti Kebangsaan Malaysia, Bangi, Malaysia

Well-written manuscripts. It is advised to double-check back any grammatical errors throughout the manuscripts. The discussion needs to be added more especially in referring to table 6 .

Competing Interests: No competing interests were disclosed.

Reviewer Expertise: Power Electronics, Power Quality, Electrical Power Engineering, Renewable Energy System, Artificial Intelligence and Agriculture-Photovoltaic.

I confirm that I have read this submission and believe that I have an appropriate level of expertise to confirm that it is of an acceptable scientific standard.

Reviewer Report 25 February 2022

https://doi.org/10.5256/f1000research.121539.r125172

(C) 2022 Paś J. This is an open access peer review report distributed under the terms of the Creative Commons Attribution License, which permits unrestricted use, distribution, and reproduction in any medium, provided the original work is properly cited.

\section{Jacek Paś}

Division of Electronic Systems Exploitations, Faculty of Electronics, Institute of Electronic Systems, Military University of Technology, Warsaw, Poland

I have studied to improve and revise the entire article again for the comments I made.

The authors have incorporated all criticisms into the revised article.

The development of the article by the authors after taking into account the comments is good. The 
article can be accepted for indexing.

The response to the reviewer's comments by the authors is very good. The authors can further extend the research on the environmental impact of high voltage lines.

Competing Interests: No competing interests were disclosed.

I confirm that I have read this submission and believe that I have an appropriate level of expertise to confirm that it is of an acceptable scientific standard.

\section{Version 1}

Reviewer Report 07 February 2022

https://doi.org/10.5256/f1000research.76690.r99796

(C) 2022 Mohd Zainuri M. This is an open access peer review report distributed under the terms of the Creative Commons Attribution License, which permits unrestricted use, distribution, and reproduction in any medium, provided the original work is properly cited.

\section{Muhammad Ammirrul Atiqi Mohd Zainuri}

Electrical, Electronic and Systems Engineering, Faculty Engineering and Built Environment, Universiti Kebangsaan Malaysia, Bangi, Malaysia

Introduction:

Add proper objectives like "this paper or manuscript present... "

Add also an overview of each section at the end of the introduction part. Methods:

Explain in more detail the flowchart of Figure 2.

Results:

Do you have any waveform that can show the EMI related to Electric field strength?

More discussion needed as referred to Table 6 in reference to each parameter discussed as in table 6.

Conclusions:

Too short, add more summary on the methods and results.

Is the work clearly and accurately presented and does it cite the current literature? Yes

Is the study design appropriate and is the work technically sound? Yes

Are sufficient details of methods and analysis provided to allow replication by others? 
Yes

If applicable, is the statistical analysis and its interpretation appropriate?

Yes

Are all the source data underlying the results available to ensure full reproducibility? Yes

Are the conclusions drawn adequately supported by the results?

Yes

Competing Interests: No competing interests were disclosed.

Reviewer Expertise: Power Electronics, Power Quality, Electrical Power Engineering, Renewable Energy System, Artificial Intelligence and Agriculture-Photovoltaic.

I confirm that I have read this submission and believe that I have an appropriate level of expertise to confirm that it is of an acceptable scientific standard, however I have significant reservations, as outlined above.

Author Response 20 Feb 2022

Chun Lim Siow, Multimedia University, Cyberjaya, Malaysia

Thank you for the invaluable comments to improve this paper. The improvements made are summarised below:

Introduction:

Add proper objectives like "this paper or manuscript present... "

The objective has been rephrased as follows:

Hence, this paper presents the simulations of the electromagnetic field radiation from various high-voltage power transmission lines in Malaysia conducted simultaneously to provide an overall comparison in terms of their corresponding EMF level as a function of distance away from the power lines.

Add also an overview of each section at the end of the introduction part.

The overview of each section has been added at the end of the introduction part. Methods:

Explain in more detail the flowchart of Figure 2.

Flowchart of Figure 2 has been explained in more detail under the Methods section. Results:

Do you have any waveform that can show the EMI related to Electric field strength? 
An additional Reference 21 has been cited to support the relation between EMI and electric field strength.

More discussion needed as referred to Table 6 in reference to each parameter discussed as in table 6 .

The discussion on Table 6 has been extended

Conclusions:

Too short, add more summary on the methods and results.

The conclusion has been expanded to be more comprehensive.

Competing Interests: No competing interests were disclosed.

Reviewer Report 07 February 2022

https://doi.org/10.5256/f1000research.76690.r120587

(C) 2022 Paś J. This is an open access peer review report distributed under the terms of the Creative Commons Attribution License, which permits unrestricted use, distribution, and reproduction in any medium, provided the original work is properly cited.

\section{Jacek Paś}

Division of Electronic Systems Exploitations, Faculty of Electronics, Institute of Electronic Systems, Military University of Technology, Warsaw, Poland

1. Please add at the end of the article a list of abbreviations used in the article.

2. The critical review of the literature on the state of the issue is done poorly.

3. Please expand the conclusions in the article, they are currently only four sentences long. Not all important graphs are included in the conclusions.

4. The article and low frequency electromagnetic field calculations around power lines are very interesting, and the results can be used to determine safety zones.

5. The issue of low-frequency electromagnetic field effects is important to the health of people living near power lines. Please refer to the $\mathrm{E}, \mathrm{H}$ component limits of standards in other countries, e.g. USA, European countries and the proposed regulations in the country.

Is the work clearly and accurately presented and does it cite the current literature? Yes

Is the study design appropriate and is the work technically sound? 
Yes

Are sufficient details of methods and analysis provided to allow replication by others? Partly

If applicable, is the statistical analysis and its interpretation appropriate?

I cannot comment. A qualified statistician is required.

Are all the source data underlying the results available to ensure full reproducibility?

Yes

Are the conclusions drawn adequately supported by the results?

Yes

Competing Interests: No competing interests were disclosed.

Reviewer Expertise: Electronic safety systems, compatibility

I confirm that I have read this submission and believe that I have an appropriate level of expertise to confirm that it is of an acceptable scientific standard, however I have significant reservations, as outlined above.

Author Response 20 Feb 2022

Chun Lim Siow, Multimedia University, Cyberjaya, Malaysia

Thank you for your invaluable comments to improve this article. The improvements made are summarised below:

1. Please add at the end of the article a list of abbreviations used in the article.

The list of abbreviations has been added at the end of the article.

2. The critical review of the literature on the state of the issue is done poorly.

The literature review has been improved. The contributions from related and cited articles have been discussed and the research gap has been clearly identified and stated.

3. Please expand the conclusions in the article, they are currently only four sentences long. Not all important graphs are included in the conclusions.

The conclusion has been expanded to be more comprehensive.

4. The article and low frequency electromagnetic field calculations around power lines are very interesting, and the results can be used to determine safety zones.

Yes, this is indeed an important issue where public awareness and understanding still has a lot of room for improvement. 
5. The issue of low-frequency electromagnetic field effects is important to the health of people living near power lines. Please refer to the $\mathrm{E}, \mathrm{H}$ component limits of standards in other countries, e.g. USA, European countries and the proposed regulations in the country.

Thank you for the recommendation. The low frequency electromagnetic field effect is indeed a very important issue for the health of people living near power lines. In this paper, we have benchmarked against the world's largest and most relevant organization namely ICNIRP, which is collaborating with many other relevant stakeholders such as World Health Organisation and national radiation protection bodies at the global level. We have also benchmarked against a regional recommendation which is European Union and two European countries (Slovenia and Italy) which sets more stringent exposure limits for the $E$ and $H$ components. For future work, it would be interesting to consider USA (with so many large states) and other European countries as per your recommendation.

Competing Interests: No competing interests were disclosed.

The benefits of publishing with F1000Research:

- Your article is published within days, with no editorial bias

- You can publish traditional articles, null/negative results, case reports, data notes and more

- The peer review process is transparent and collaborative

- Your article is indexed in PubMed after passing peer review

- Dedicated customer support at every stage

For pre-submission enquiries, contact research@f1000.com 